\title{
Heat Transfer by Porous Pin Fins and Nanofluid in Rectangular Minichannels
}

\author{
K. S. ARJUN*, K. RAKESH** \\ *Department of Mechanical Engineering, Indian Institute of Technology (ISM), Dhanbad-826004, India, \\ E-mail:arjun@mece.ism.ac.in \\ **Department of Mechanical Engineering, Indian Institute of Technology (ISM), Dhanbad-826004, India \\ crossref http://dx.doi.org/10.5755/j01.mech.24.1.17284
}

\section{Introduction}

Porous media has two specifications: First its dissipation area is greater than the conventional fins that enhance heat convection. Second the irregular motion of the fluid flow around the individual beads mixes the fluid more effectively. Nanofluids are mixtures of base fluid with a very small amount of nanoparticles having dimensions from 1 to $100 \mathrm{~nm}$, with very high thermal conductivities, so it would be the best convection heat transfer by using porous media and nanofluids. Thus studies for the enhancement of convective heat transfer involving nanofluids in porous media are important.Rapid heat removal from heated surfaces and reducing material weight and cost become a major task for design of heat exchanger equipments like Cooling of Internal Combustion engines. Development of super heat exchangers requires fabrication of efficient techniques to exchange great amount of heat between surface such as extended surface and ambient fluid.

Pin fins have a variety of applications in industry due to their excellent heat transfer performance, e.g., in cooling of electronic components, in cooling of gas turbine blades, and recently, in hot water boilers of central heating systems, etc. [1]. In the two early studies by Sahiti et al. [2, $3]$, it was demonstrated that pin fin arrays offer the most effective way of enhancing the heat transfer rate within a particular heat exchanger volume. However, the pressure drops in such heat exchangers are usually much higher than those in others [1]; this defect greatly lowers the overall heat transfer performances of pin fin heat exchangers and as a result, their applications are restricted. In order to reduce the pressure drops and improve the overall heat transfer performances for pin fin heat exchangers, porous metal pin fin arrays may be used instead of traditional solid metal pin fin arrays.

As porous media can significantly intensify the mixing of fluid flow and increase the contact surface area with fluid inside, it has been regarded as an effective way to enhance heat transfer by using porous media [4]. The flow and heat transfer in porous pin fin heat exchangers for present study can be modeled as forced convective heat transfer in partially filled porous channels. The researches on forced convection with partially filled porous configurations have been investigated extensively in the last years. Hadim [5] studied the laminar forced convection in a fully or partially filled porous channel containing discrete heat sources on the bottom wall. The Brinkman- Forchheimer [6] found that when the width of the heat source and the space between the porous layers were of same magnitudes as the channel height, the heat transfer enhancement in the partially filled channel was almost the same as that in the fully filled porous channel while the pressure drop was much lower.

Hadim and Bethancourt [7] later studied the similar problem in a partially filled porous channel. They found that when the heat source width was decreased, there was a moderate increase in heat transfer enhancement and a significant decrease in pressure drop. Huang and Vafai [8] presented a detailed investigation of forced convection in a channel filled with multiple emplaced porous blocks. With comparison of the local Nusselt number distributions between the channel with and without porous blocks, they found that significant heat transfer augmentation can be achieved through the emplacement of porous blocks. Huang et al. [9] later presented a similar investigation in cooling of multiple heated blocks covered with porous media. The results showed that significant cooling augmentation of the blocks can be achieved through the cover of finite-sized porous substance. Other similar studies of forced convection in a channel filled with porous blocks can also be found in References. [10, $11]$.

This interest is primarily due to the fact that this kind of structure is encountered in many engineering applications such as drying processes, filtration, thermal insulation, geothermal systems, ground water and oil flow, as well as heat exchangers in all types. Flows with porous media occur in power stations of many practical engineering applications where heating or cooling is required. Some applications include cooling electronic equipment, cooling turbines blades, combustion systems, chemical processes, high performance heat exchangers, and energy systems equipment. The mixing of the high and low energy fluids which occurs in these applications significantly influences the performance of these devices. One of the ways to enhance heat transfer is to employ porous media with and without nanofluid. Porous media is a material containing pores such as metals and oxides. These pores are typically filled with a fluid (liquid or gas). Thus, it causes increase in heat transfer in the flow field. Past studies showed that porous media and nanofluid exhibit enhanced thermal properties, such as higher thermal conductivity and convective heat transfer coefficients compared to the base material.

Kosar et al. [12] experimentally studied the pressure drop and thermal performance of de-ionized water at Reynolds numbers up to 112 over a bank of shrouded staggered micro-pin-fins. They found that for Reynolds numbers above 50, the long tube correlations predicted the heat transfer coefficients well but for Reynolds numbers below w50 the existing correlation over predict the experimental Nusselt number. They reported that endwalls effect is the 
primary cause for this anomaly. Prasher et al. [13] experimentally investigated the hydraulic and thermal performances of low aspect ratio micro-pin-fin cold plates with square and circular cross-sections under cross flow conditions. They found that some type of transition is happening around $R e=100$, therefore, they suggested turbulent flow behavior in micro-pin-fin cold plates for $R e>100$.

Koo and Kleinstreuer [14] conducted a numerical study to analyze the convection heat transfers of nanofluids in a rectangular microchannel $(300 \mathrm{~mm} \hat{\mathrm{A}} 50 \mathrm{~mm})$. Their results indicated higher Nusselt number for ethylene glycolbased nanofluids than water-based nanofluids. Li and Kleinstreuer [15] numerically studied the thermal performance of a trapezoidal microchannel with nanofluid as working fluid. Their results showed that the nanofluid enhances both pumping power and thermal performance and with increasing the volume fraction, the thermal performance increases. Recently, Seyf and Mohammadian [16] numerically studied the thermal performance of a counter flow microchannel heat exchangers. They reported that the nanofluid enhances both pumping power and thermal performance and increasing the volume fraction leads to increasing thermal performance of system.

The heat transfers and pressure drop effect of the triangular pin-fin shape on the overall performance of the carbon nanotube bundles as $3 \mathrm{D}$ porous micro pin-fins with staggered arrangement is studied using FLUENT 15.0, compared to solid medium. The thermal performance in terms of Nusselt number of the $\mathrm{CuO} / \mathrm{H} 2 \mathrm{O}$ and $\mathrm{Al} 2 \mathrm{O} 3 / \mathrm{H} 2 \mathrm{O}$ nanofluids at different concentrations from 0.001 to $1 \%$ on a rectangular minichannel surface with multi-walled nanotube fins, in comparison with water is analyzed for Re 100 to 2000 in the present study. $\mathrm{Nu}$ and pressure drop across the finned structure were validated with the experimental work.

\section{Device geometry and computational modelling}

Staggered triangle device geometries were developed and used for this simulation. A $1 \mathrm{~mm}$ thick rectangular minichannel made of silicon with a size of $45 \times 15 \mathrm{~mm}$ is used. Carbon Multi Walled Nano Tubes (MWNTs) with broad diameter distribution of $10-100 \mathrm{~nm}$ are used. Nanotubes with a diameter of $0.5 \mathrm{~mm}$ and $0.75 \mathrm{~mm}$ in height were grown at the center of the silicon wafer at the bottom in a rectangular area of $25 \times 15 \mathrm{~mm} 2$. Figure 1 is the device geometry of the minichannel including triangular staggered micro pin fins and their dimensions.

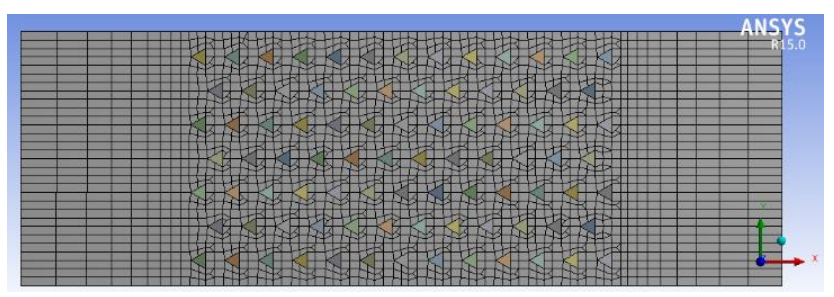

Fig. 1 Minichannel model with Triangular Carbon Nano Tube pin fins in a staggered array

For these simulations the flow regime is considered to be a continuum flow. The flow through this model is solved using the Navier-Stokes equations. The basic governing equations for a steady-state, incompressible flow are:
Continuity equations:

$(\nabla \cdot \rho u)=0$

Conservation of momentum:

$\rho(u \cdot \nabla u)=-\nabla p+\mu \nabla^{2} u$.

Conservation of energy:

$\rho c_{p}(u \cdot T u)=\nabla^{2} T$.

The geometry is meshed based on the finite volume method. FLUENT 15.0 [17] is used to model the flow past the pin fins in a staggered array in the rectangular minichannel and solves the governing equations iteratively for each control volume. The convergence criterion for the solution is residuals of less than 10-6 for both continuity and momentum. Each model contained a fine mesh surrounding the finned section with a less dense mesh as the channel extends to the inlet and outlet. Fine mesh is developed such that the mass flow rate no longer changes with increase of number of mesh elements and the results converge with higher accuracy, while considering computational cost as well. In order to optimize the micro pin fins; solid and porous material properties are applied to the pin finned geometry. A constant heat flux is applied to the bottom of the channel. The flow is ensured hydrodynamically developed before the fluid reaches the heated region.

The transverse and longitudinal spacing of the fins for this study is equal to double the width of the fin. For all geometries, the fin material property used is that of Carbon Nano Tubes (CNTs); however, because the thermal conductivity is unknown when nanotubes are clustered together to form fins, an effective thermal conductivity of $400 \mathrm{~W} / \mathrm{mK}$ is used for solid medium [18]. This study was simulated using heat flux and multiple flow rate inputs. Initial inlet temperature and outlet static pressure values applied to the model are assumed for all simulations to be $25^{\circ} \mathrm{C}$ and $0 \mathrm{~Pa}$, respectively. To monitor the heat transfer coefficient and the heat transfer rate, the outer walls of the channel are set to be adiabatic. No-slip boundary conditions and no interfacial resistance are assumed at the wall/fluid interface. Water is used as the working fluid flowing through this heat exchanger with different velocities through the inlet of the channel. These simulations are in the single phase regime and fluid properties are kept constant throughout the simulations. Water flows past pin fins carrying heat subjected by bottom surface. A constant heat flux of $100 \mathrm{~W} / \mathrm{m}^{2}$ is applied to a $15 \times 25 \mathrm{~mm}^{2}$ area at bottom of the channel.

With constant heat flux, different media of nanotube bundles and forced convection, the temperature across surface of the microchip and bulk temperature of fluid vary. To accurately obtain heat transfer coefficient, $h$, across the microchip region for simulations, an average $h$ was obtained. There were some uncertainties within the model that can increase the error in the approximation. No slip boundary conditions are used to define these simulations at fluid solid interfaces. As the scales start moving toward micro or nano regime, conventional continuum calculations can no longer be used. Also, resistance created at interface of CNTs and working fluid is still a major issue. In this model, no 
interfacial resistance is applied. The CNT fins are modeled as a solid emerging out from surface rather than bundles of nanotubes. CNT micro pin fins are made up of many nanotubes where the fluid penetrates through small gaps, or nanochannels, allowing for an increased in convection within the system. As shown by Shenoy et al. [19], CNTs absorb the fluid at high temperatures creating a porous like material and increases heat transfer due to a hastened nucleate boiling onset initiating phase change. This assumption in the model may underestimate the thermal performance of CNT solid fins. Also, there are ongoing investigations of the effective thermal conductivities of CNT bundles; therefore, an estimation of an effective thermal conductivity is used. Hence, the case is simulated using porous medium. Influence of $\mathrm{Al}_{2} \mathrm{O}_{3} / \mathrm{H}_{2} \mathrm{O}$ and $\mathrm{CuO} / \mathrm{H}_{2} \mathrm{O}$ nanofluids instead of water on the thermal performance in comparison with cases of solid nanotubes and with porous nanotube fins are also investigated at volumetric concentrations of 0.001 to $1 \%$.

A porous media is used to enhance a heat flux removal by increasing the heat transfer area between solid and fluid regions and good thermal contact between each porous particle. Thermal parameters increase when the pore size decreases. A decrease in pore diameter increases the pressure drop across the porous medium. ANSYS FLUENT uses and reports a superficial velocity inside the porous medium, based on the volumetric flow rate, to ensure continuity of the velocity vectors across the porous medium interface. The effect of the porous medium on the turbulence field is approximated. FLUENT assumes that the porosity is isotropic and can vary with space and time; assumes thermal equilibrium between the porous media solids and multiphase fluid flows. The solids temperature is thus estimated by phase temperatures. FLUENT solves the standard energy transport equation in porous media regions with modifications to the conduction flux and the transient terms only. In the porous medium, the conduction flux uses an effective conductivity, computed as the volume average of the fluid and solid conductivity, via user-defined functions. The transient term includes the thermal inertia of the solid region on the medium. Set the viscous and inertial resistance coefficients are set, the direction vectors are defined for which they apply and the porosity of the porous medium is specified.

\section{Results and discussion}

Average $R e$ and $\mathrm{Nu}$ are collected for the different inputted heat fluxes and flow rates. Average axial velocity was obtained as the fluid flowed across the fin bank:

$$
R e=\frac{\rho u D_{c}}{\mu} .
$$

$\mathrm{Nu}$ is proportional to the average heat transfer coefficient, $h$, and the hydraulic diameter of the channel, and non-proportional to the thermal conductivity, $k$.

$$
N u=\frac{h D_{c}}{k} .
$$

The average heat transfer coefficient is obtained by the amount of surface area of the fins and the base that the fluid interacts with. The expression is given below:

$$
h=\frac{\left(h_{f i n} A_{f i n}+h_{b} A_{b}\right)}{\left(A_{f i n}+A_{b}\right)},
$$

where: $h_{f i n}$ and $h_{b}$ are the heat transfer coefficients of the fin and base of the heated region only; $A_{\text {fin }}$ and $A_{b}$ are the surface areas in which the fluid touches the fin and heated base region, respectively. The heat transfer coefficient of the fin and base at the fluid interface is obtained by:

$$
h=\frac{q}{T_{w}-T_{n w}},
$$

where: $q$ is the wall heat flux, $T_{w}$ is the temperature of the wall and $T_{n w}$ is the near wall temperature. Pressure drop is determined across the pin fins only to avoid any entrance and exit effects of the fluid flow using a maximum axial velocity. The results for all of the studies are given below.

\subsection{Solid and porous media pin fins}

For single phase flows, changing solid medium to porous medium properties show $6 \%$ higher variation within the Nusselt number and pressure drop values. The results portraying this study are shown in Fig. 2.

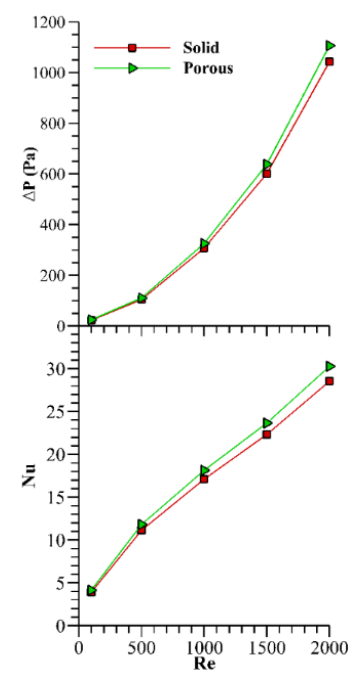

Fig. $2 \mathrm{Nu}$ and pressure drop versus $R e$ for Solid and Porous media Pin fins

These findings show that the chosen porous fin medium had little effect on the Nusselt number values. In this study, CNTs are modeled as a solid fin but in reality the fins are more of a porous media that contain small gaps where the fluid can penetrate. The nanotubes can also initiate nucleation sites initiating boiling and therefore enhancing heat transfer. Because of this, the results for the CNT fins are underestimated using solid medium. From this conclusion, the CNTs yield a greater thermal performance than the other fins when simulated as porous medium. Pressure drops display similar difference between the fin media. Pressure drop for these studies have shown critical in optimizing fin geometry and topology. For a single phase, laminar, flow through a minichannel, $\mathrm{Nu}$ values for porous pin fins did show improvement compared to the solid fin channel. As Reynolds number increases from 100 to 2000, there is a 
drastic increase in Nusselt number and pressure drop values to the tune of $628 \%$.

\subsection{Effect of use of different nanofluids}

The porous MWNT device has a thermal increase of about $88 \%$ using $0.01 \%$ volume concentration of $\mathrm{Al}_{2} \mathrm{O}_{3}$ nanofluid than the same device using de-ionized water and $38 \%$ increase with respect to 0.001 volume concentration (Fig. 3). Increase in Reynolds numbers from 100 to 2000 showed increase in Nusselt number values in all cases of use of nanofluids. The finned MWNTs devices using $1 \% \mathrm{Al}_{2} \mathrm{O}_{3}$ nanofluid had decreases of $77 \%$ and $40 \%$ pressure drop respectively, for the same base temperature compared to solid MWNTs with no nanofluid in the channel and 0.01 volumetric concentration. Similarly, 91\% thermal increase for staggered triangular solid fins with $0.01 \%$ volume concentration of $\mathrm{CuO}$ nanofluid in place of using water and $80 \%$ increase in place of use of porous fins could be noticed (Fig. 4).

The pressure drop obtained using the nanofluid for each channel show lower values with the addition of nano particles in place of solid or porous pin fins. The MWNTs

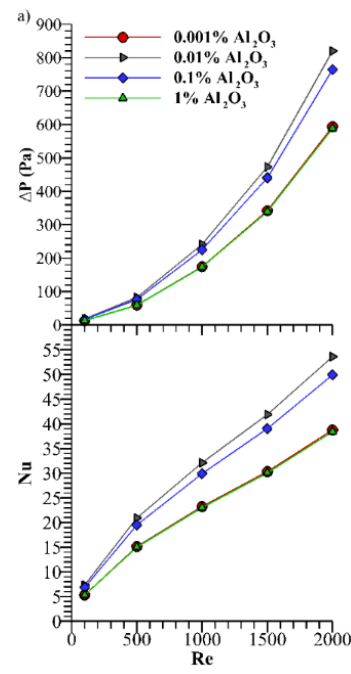

Fig. 3 Effect of use of different volumetric concentrations of $\mathrm{Al}_{2} \mathrm{O}_{3}$ /water nanofluid

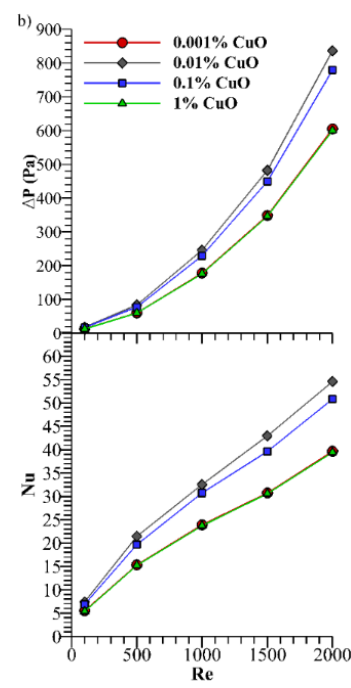

Fig. 4 Effect of use of different volumetric concentrations of $\mathrm{CuO} /$ water nanofluid device caused lower pressure drops with $0.1 \%$ and $1 \%$ volumetric concentrations of both $\mathrm{Al}_{2} \mathrm{O}_{3}$ nanofluid and $\mathrm{CuO}$ nanofluid when compared to the $0.01 \%$ volumetric concentration (39\%). Higher pressure drops were also noticed for $0.01 \%$ volumetric concentrations of $\mathrm{Al}_{2} \mathrm{O}_{3}$ nanofluid and $\mathrm{CuO}$ nanofluid and porous medium in comparison to $0.001 \%$ volumetric concentrations $(38 \%) . \mathrm{Al}_{2} \mathrm{O}_{3}$ nanofluid registered $77 \%$ and $88 \%$ lower pressure drops when compared to solid and porous pin finned channels without nanofluids. Increase in Reynolds numbers from 100 to 2000 showed increase in pressure drops in all cases of use of nanofluids.

This study verifies that the major enhancement, when using nanofluids to cool heated surfaces, is the surface defects that are caused by deposits on the surface. As stated by other researchers [20], these particles create imperfections on the surface causing an increased wettability. The channels which already contain engineered structures and porous in nature increase the surface area and wettability and the thermal performance is significantly improved.

\section{Validation}

To validate, experimental results taken from work completed by Liu et al. [21] for a heat sink containing 625 square micro pin fins of $445 \times 445 \mu \mathrm{m}^{2}$ in a staggered array was solved with water used as the working fluid flowing with Re ranging from 60 to 800 . The fins and channel have a height of $3 \mathrm{~mm}$. The longitudal and transverse spacing of the fin measured $565.7 \mu \mathrm{m}$. The ambient fluid temperature is initally at room temperature and heat is applied to the bottom surface. The $N u$, and pressure drop, across the finned structure were calculated and compared to the experimental data, shown in Fig. 8 and are very similar to the value obtained in the experimental work for $\mathrm{Nu}$ values and pressure drop except for $\operatorname{Re} 100$ and $\operatorname{Re} 500$ (for pressure drop alone) within $8.3-32.8 \%$. The disturbance environment of flow can cause variations in the velocity field and this might be the possible difference in experimental data results of pressure drop at $\operatorname{Re} 100$ with respect to that of numerical data results. The difference in data at $R e=500$ is within the acceptable limits.

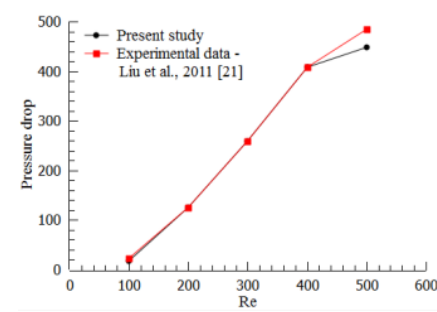

a

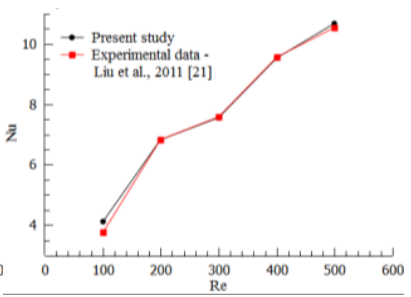

Fig. 5 Validation of simulated data of Staggered Square porous pin fins with experimental data [21] at different $R e:$ a. $N u$ b. Pressure drop

\section{Conclusions}

We found that the staggered porous triangular pin fin shape yields the higher $N u$ and pressure drop at all $R e$ studied, ie. from 100 to 2000, in comparison to solid pin-fin heat sinks. The effects of $\mathrm{CuO} / \mathrm{H}_{2} \mathrm{O}$ nanofluid, on $\mathrm{Nu}$ and pressure drop were better in comparison to $\mathrm{Al}_{2} \mathrm{O}_{3} / \mathrm{H}_{2} \mathrm{O}$ 
nanofluid. The results showed that in all $R e$, adding low volume fraction of nanoparticles $(0.001 \%$ and $0.01 \%)$ to the base fluid leads to significant increase of $N u$ and decrease of pressure drop up to $0.01 \%$, but this trend is reversed for higher volume fractions of $0.1 \%$ and $1 \%$. As $R e$ increases, $\mathrm{Nu}$ and pressure drop also increases at a particular volume fraction of both nanofluids as well as for porous and solid micro-pin- fins used in rectangular mini channel. The following conclusions can be drawn from the results:

- Both $\mathrm{Nu}$ and pressure drop values of staggered triangle porous micro-pin-fins registered $6 \%$ higher in comparison to similar solid fins.

- $\mathrm{Al}_{2} \mathrm{O}_{3} / \mathrm{H}_{2} \mathrm{O}$ nanofluid in comparison to the minichannel containing no nanofluid and pin fins as solid and porous medium recorded $88 \%$ and $77 \%$ higher $N u$ values. The associated pressure drops are seen decreased; but in reverse magnitude.

- $\mathrm{CuO} / \mathrm{H}_{2} \mathrm{O}$ nanofluid yields better thermal performance than $\mathrm{Al}_{2} \mathrm{O}_{3} / \mathrm{H}_{2} \mathrm{O}$ nanofluid in comparison to the minichannel containing no nanofluid with pin fins both as solid $(91 \%)$ and porous medium $(80 \%)$. Pressure drop decreased with a $74 \%$ and $84 \%$ differential to the solid and porous pin finned mini channels respectively.

- Both $\mathrm{Al}_{2} \mathrm{O}_{3} / \mathrm{H}_{2} \mathrm{O}$ and $\mathrm{CuO} / \mathrm{H}_{2} \mathrm{O}$ nanofluids registered a $38 \%$ higher $\mathrm{Nu}$ from 0.001 to $0.01 \%$ volumetric concentrations and then decreases at 0.1 and $1 \%$ volumetric concentrations so that there exist no significant difference in $\mathrm{Nu}$ values at 0.001 and $1 \%$ volumetric concentrations. The associated pressure drops are seen decreased at $0.001 \%$ volumetric concentrations; then become the highest among the tested volumetric concentrations of similar magnitude as that of $N u$ (but lower than no nanofluid cases) and then decreases sequentially at 0.1 and $1 \%$ volumetric concentrations.

- $\quad N u$ and pressure drop, across the square finned structure were calculated and are found very similar to the value obtained in the experimental work for $N u$ values and pressure drop except for $\operatorname{Re} 100$ and $\operatorname{Re} 500$ (for pressure drop alone) within $8.3-32.8 \%$.

\section{References}

1. Sahiti, N.; Lemouedda, A.; Stojkovic, D.; Durst, F.; Franz, E. 2006. Performance Comparison of Pin Fin InDuct Flow Arrays with Various Pin Cross-Sections, Appllied Thermal Engineering 26: 1176-1192.

http://dx.doi.org/10.1016/j.applthermaleng.2005.10.042

2. Sahiti, N.; Durst, F.; Dewan, A. 2005. Heat Transfer Enhancement by Pin Elements, International Journal of Heat and Mass Transfer 48: 4738-4747.

http://dx.doi.org/0.1016/j.ijheatmasstranfer.2005.07.001.

3. Sahiti, N.; Durst, F.; Dewan, A. 2006. Strategy for Selection of Elements for Heat Transfer Enhancement, International Journal of Heat and Mass Transfer 49: 33923400 .

http://dx.doi.org/10.1016/j.ijheatmasstransfer.2006.03. 011.

4. Jiang, P. X.; Li, M.; Lu, T. J.; Yu, L.; Ren, Z. P. 2004. Experimental Research on Convection Heat Transfer in Sintered Porous Plate Channels, International Journal of Heat and Mass Transfer 47: 2085- 2096.

http://dx.doi.org/10.1016/j.ijheatmasstransfer.2003.12.0
04.

5. Hadim, A. 1994. Forced Convection in a Porous Channel with Localized Heat Sources, ASME Journal of Heat Transfer 116: 465-472. http://dx.doi.org/10.1115/1.2911419.

6. Brinkman, H. C. 1947 . On the permeability of media consisting of closely packed porous particle, Journal of Applied Sciences Research 1: 81-86. http://dx.doi.org/10.1007/BF02120318.

7. Hadim, A.; Bethancourt, A. 1995. Numerical Study of Forced Convection in a Partially Porous Channel with Discrete Heat Sources, ASME Journal of Electronic Packaging 117: 46-51. http://dx.doi.org/10.1115/1.2792066.

8. Huang, P. C.; Vafai, K. 1994. Analysis of Forced Convection Enhancement in a Channel Using Porous Blocks, Journal of Thermophysics and Heat Transfer 8: 563-573. http://dx.doi.org/10.2514/3.579.

9. Huang, P. C.; Yang, C. F.; Hwang, J. J.; Chui, M. T. 2005. Enhancement of Forced-Convection Cooling of Multiple Heated Blocks in a Channel Using Porous Covers, International Journal of Heat and Mass Transfer 48: 647-664.

http://dx.doi.org/doi:10.1016/j.ijheatmasstransfer.2004. 07.041 .

10. Fu, W.; Huang, H.; Lion, W. 1996. Thermal Enhancement in Laminar Channel Flow with a Porous Block, International Journal of Heat and Mass Transfer 39: 21652175 .

http://dx.doi.org/10.1016/0017-9310(95)002081.

11. Ould-Amer, Y.; Chikh, S.; Bouhadef, K.; Lauriat, G. 1998. Forced Convection Cooling Enhancement by Use of Porous Materials, International Journal of Heat and Fluid Flow 19: 251-258.

http://dx.doi.org/10.1016/S0142-727X(98)000046.

12. Kosar, A.; Peles, Y. 2006. Thermal-hydraulic performance of MEMS-based pin fin heat sink, Journal of Heat Transfer 128 (2): 121-131.

http://dx.doi.org/10.1115/1.2137760.

13. Prasher, R.S.; Dirner, J.; Chang, I.-Y.; Myers, A.; Chau, D.; He, D.; Prstic, S.; Pfefferkorn, F. 2007. Nusselt number and friction factor of staggered arrays of low aspect ratio micropin-fins under cross flow for water as fluid, Journal of Heat Transfer 129: 141-153. http://dx.doi.org/10.1115/1.2402179.

14. Koo, J.; Kleinstreuer, C. 2005. Laminar nanofluid flow in micro heat-sinks, International Journal of Heat and Mass Transfer 48: 2652-2661.

http://dx.doi.org/10.1016/j.ijheatmasstransfer.2005.01.029.

15. Li, J.; Kleinstreuer, C. 2008. Thermal performance of nanofluid flow in microchannels, International Journal of Heat and Fluid Flow 29: 1221-1232.

http://dx.doi.org/10.1016/j.ijheatfluidflow.2008.01.005.

16. Seyf, H.R.; Mohammadian, S.K. 2011. Thermal and hydraulic performance of counterflow microchannel heat exchangers with and without nanofluids, Journal of Heat Transfer 133: 81801-81809. http://dx.doi.org/10.1115/1.4003553.

17. Ansys. 2013. ANSYS Fluent 15.0 Theory Guide, ANSYS Inc., Canonsburg, PA, USA, pp 1-780.

18. Zhong, X.; Fan, Y.; Liu, J.; Zhang, Y.; Wang, T.; Cheng, Z. 2007. A Study of CFD Simulation for On- 
chip Cooling with 2D CNT Micro-Fin Array, Proceedings of the International Symposium on High Density packaging and Microsystem Integration, 7: 1-6.

http://dx.doi.org/10.1109/HDP.2007.4283649.

19. Shenoy, S.; Tullius, J. F.; Bayazitoglu, Y. 2011. Minichannels with Carbon Nanotube Structured Surfaces for Cooling Applications, International Journal of Heat and Mass Transfer 54 (25-26): 5379-5385.

http://dx.doi.org/10.1016/j.ijheatmasstransfer.2011.08.0 05.

20. Ahn, H. S.; Kim, M. H. 2012. A Review on Critical Heat Flux Enhancement with Nanofluids and Surface Modification, Journal of Heat Transfer 134 (2): 024001. http://dx.doi.org/10.1115/1.4005065.

21. Liu M.; Liu D.; Xu S.; Chen Y. 2011. Experimental Study on Liquid Flow and Heat Transfer in Micro Square Pin Fin Heat Sink, International Journal of Heat and Mass Transfer 54 (25-26): 5602-5611.

http://dx.doi.org/10.1016/j.ijheatmasstransfer.2011.07.0 13.
K. S. Arjun, K. Rakesh

HEAT TRANSFER BY POROUS PIN FINS AND
NANOFLUID IN RECTANGULAR MINICHANNELS

S u m m a r y

The forced convective heat transfers with $3 \mathrm{D}$, triangular, porous pin fins as bundle of MWCNTs at the bottom of rectangular mini channels made of silicon. Overall heat transfer performances in porous pin fins are much better than those in traditional solid pin fins. As nanofluid volume concentration increases, pressure drops and heat fluxes in porous pin fin channels increase and maximal overall heat transfer are obtained at $0.01 \%$ concentration. Enhancement in $\mathrm{Nu}$ were $6 \%$ with respect to porosity and $88 \%$ with respect to $0.01 \% \mathrm{Al}_{2} \mathrm{O}_{3} / \mathrm{H}_{2} \mathrm{O}$. With the same physical parameters, the $\mathrm{Nu}$ in the staggered triangular porous pin fin channels of CNTs with fin height of $0.75 \mathrm{~mm}$, smaller fin width of $0.5 \mathrm{~mm}$ and spacing double the fin width using $0.01 \%$ $\mathrm{CuO} / \mathrm{H}_{2} \mathrm{O}$ nanofluid showed the highest enhancement of $91 \%$ at higher $R e$ with a corresponding decrease in pressure drop of $25 \%$.

Keywords: porous pin fin, minichannel, heat transfer, nanofluid, carbon nanotube.

Received December 18, 2016

Accepted February 15, 2018 